\title{
A non-linear deformation theory applied to two dimensional pretensioned cable assemblies
}

\section{H. A. BUCHHOLDT}

\section{Dr T. J. Poskitt, University of Manchester}

This Paper presents an interesting procedure for analysing plane assemblies of cables undergoing finite displacements under load.

62. Since the problem being treated is one of finite strain the virtual work equation should be used in its infinitesimal form and not the finite form which is adopted in equation (3). Using the notation of the Paper, let $x$ be the displacement vector of the joints and $e$ the elastic deformation vector of the links. Then if small variations of these $d x$ and de are taken a compatible set of virtual displacements is obtained, and equation (3) becomes

$$
P^{T} d e=F^{T} d x
$$

63. In an unnumbered equation in $\S 33$

$$
\left(L_{j n}+e_{j n}\right)^{2}=\sum_{i}\left(\left(X_{n i}-X_{j i}\right)+\left(x_{n i}-x_{j i}\right)\right)^{2}
$$

is given for the deformed state of link $j n$. Expanding this expression gives

$$
2 L_{j n} e_{j n}+e_{j n}^{2}=\sum_{i}\left(2\left(X_{n i}-X_{j i}\right)\left(x_{n i}-x_{j i}\right)+\left(x_{n !}-x_{j i}\right)^{2}\right)
$$

$e_{j n}{ }^{2}$ is then ignored on the grounds that it is of the second order of smallness. To be consistent with this the $\left(x_{n i}-x_{j i}\right)^{2}$ term should also be ignored since it also is of the second order of smallness. No additional accuracy results from retaining $\left(x_{n i}-x_{j i}\right)^{2}$ and ignoring $e_{j n}{ }^{2}$. The simplification which results in, for example, the calculation of $a_{1} a_{2}$ etc, would make it appear desirable to ignore $\left(x_{n t}-x_{j i}\right)^{2}$.

64. An important cost factor in the computer solution of a non-linear structure of the type under consideration is the convergence of the iteration formula given in equation (19)

$$
\left\{x_{(k+1)}\right\}=\left\{x_{(k)}\right\}+S\left\{v_{(k)}\right\}
$$

For this to converge absolutely, i.e. for the left and right hand sides ultimately to generate an equality, then

either

$$
\left\{v_{(k)}\right\} \rightarrow 0
$$

or

$$
S \rightarrow 0
$$

65. Now $\left\{v_{(k)}\right\}$ is defined as the vector which is obtained by dividing each element in the gradient vector $\left\{\partial W / \partial x_{(k)}\right\}$ by $-R$, thus

$$
\left\{v_{(k)}\right\}=-\left\{\partial W / \partial x_{(k)}\right\} / R
$$

The gradient vector represents the unbalanced forces or residuals acting at the joints. As the process converges these residuals tend to zero, i.e.

$$
\left\{\partial W / \partial x_{(c)}\right\} \rightarrow 0
$$

As a consequence of this

$$
R=\left(\left\{\partial W / \partial x_{(k)}\right\}^{T}\left\{\partial W / \partial x_{(k)}\right\}\right)^{1 / 2} \rightarrow 0
$$

Paper published: Proc. Instn civ. Engrs, 1969, 42 (Jan.) 129-141. 
as stated in $\$ 41$. From equation (32) it will be seen that if the process is converging then $\left\{v_{(k)}\right\}$ is a vector whose elements tend to the indeterminate form $0 / 0$. This type of situation can be expected to give numerical difficulties.

66. Due to the indeterminate nature of the elements of $\left\{v_{(k)}\right\}$, if convergence is taking place it must be as a result of the second criterion given in equation (31). With a decreasing value of $S$, a stage will ultimately be reached where its determination from equation (21) is given very closely by

$$
2 C_{3} S+C_{4}=0
$$

This is clearly true since as $S \rightarrow 0$ terms of second and higher orders in $S$ become negligible. For equation (33) to give a small value of $S$ then $C_{4}$ must be small. Thus to obtain a diminishing value of $S$, which is required if the process is to converge, then $C_{4} \rightarrow 0$. Now $C_{4}$ is a function of the vector $\left\{v_{(k)}\right\}$ and since this tends to become indeterminable as the process converges, $C_{4}$ cannot be guaranteed to tend to zero.

67. It therefore appears that the iteration formula given in equation (19) is inherently unstable. A practical demonstration of this is given in the following example where after the method has given answers which are $99.99 \%$ accurate in its first cycle, it then proceeds to go unstable.

68. The structure shown in Fig. 9 is composed of flexible cables prestressed between rigid anchorages at $B, C$ and $D$. The pretensioning forces in $A C$ and $A D$ are $P_{0}$ and in $\mathrm{AB}$ is $\sqrt{ } 2 P_{0}$. The values of $E A$ (Young's modulus $\times$ cross-sectional area $A$ ) are indicated. Loading is applied to joint $\mathrm{A}$, and in Table 2 the results are given for cases when $g=1$ and 2 .

69. The results shown in Table 2 are especially interesting for the case when $g=1$. As mentioned earlier, for this case the first cycle of iteration gives answers with an error of less than $0.01 \%$, but, despite this very close approach to the exact answer, the second and third iterations show divergence due to the inherent instability of the method. By the 25 th cycle of iteration the result of instability has been to magnify the errors until the deflexions are $10^{8}$ greater than they should be.

70. In the second case when the applied loading vector is $\{2 W, W\}$, the deflexions after the first cycle of iteration are not as good approximations as they were in the first case. Apart from this difference, the growth of the instability shows a pattern similar to the first case.

71. The use of the Ritz method with a single mode of the type used in the Paper has advantages if hand calculation of problems is necessary. However, if a digital

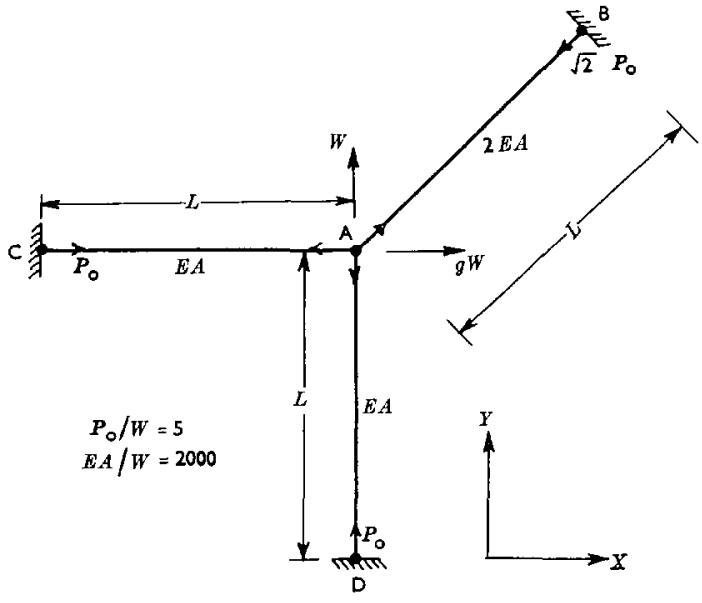

Fig. 9 
computer is available a multidegree of freedom solution is better. Each actual degree of freedom of the structure can then be varied simultaneously, and the result might be expected to converge more rapidly than in the case where several degrees of freedom have been reduced to a single "representative' displacement. This proves to be the case with the test problem which has been solved by the Newton-Raphson method. ${ }^{7}$ This converges to the final answer in two cycles of iteration. The technique of applying this method to structures in general has been considered elsewhere. ${ }^{8}$ For the present test problem it was applied directly to equations (17), and the variables $x$ and $y$ were solved for simultaneously. The results are given in Table 2 .

72. A number of general frame analysis programs are now available on different makes of computer which will solve as special cases cable net problems of the type considered here.

73. In equation (25) the initial strain energy $U_{0}$ is included but was not present in the parent equation (22). Since equation (22) is concerned with the change in strain energy due to the displacement $e_{y_{n}}$ and not its absolute magnitude, for consistency equation (25) should also be for changes in strain energy, and the term $U_{0}$ must not then be included.

\section{Mr C. W. Brown, Freeman Fox \& Partners}

The Author has presented a novel and elegant method of analysing two dimensional pretensioned cable assemblies. I think he may have been somewhat over-hasty in his dismissal of existing methods and, as will be shown later, satisfactory results can be obtained using a more conventional approach. Nevertheless, the Author's method appears to have two substantial advantages. The first is its efficiency; a check on a simple structure has indicated that convergence should be very rapid, and I should like to know how many cycles of iteration were required to yield errors of less than, say, $1 \%$ and $0.01 \%$ in the example quoted in the Paper for $W=10000 \mathrm{lbf} /$ joint. The second major advantage lies in the unexacting demands the method appears to make on computer hardware: using it, a machine with a small store and working to a comparatively low number of significant figures should be able to analyse quite complex structures.

75. The main disadvantage of the method as described lies in its inability to include flexural members. Structures consisting of assemblies of beams and pretensioned cables are not uncommon-the stiffened suspension bridge is an obvious example-and I am interested to know if the Author has attempted to extend the method to include beams.

76. Some time ago, I wrote a program for general finite deflexion analysis of rigid jointed plane frames, and I have used it to analyse the Author's example by putting the second moment of area of all members equal to zero. Some results are quoted.

77. The method used is based on that described by Saafan and Brotton ${ }^{9}$ and is an iterative stiffness process, each cycle of which consists in principle of the following steps:

(a) compute the unbalanced forces at each joint and stop if these are negligible, otherwise

(b) compute the incremental displacements due to $(a)$ by means of a linear stiffness analysis and add them to the current values at the beginning of the cycle;

(c) compute member forces from (b) and output results if required;

(d) return to $(a)$.

78. The method as described, however, is for the analysis of structures; in order to make it converge for pretensioned structural mechanisms, it is necessary to use the 'tangent stiffness matrix' in the linear analyses. This matrix, in which the terms are dependent on the current displacements of the structure, has been derived in detail by Tezcan ${ }^{10}$ by considering a generalized Newton-Raphson process for solving the nonlinear structural equations. If a number of small order flexural terms are omitted, 
DISCUSSION

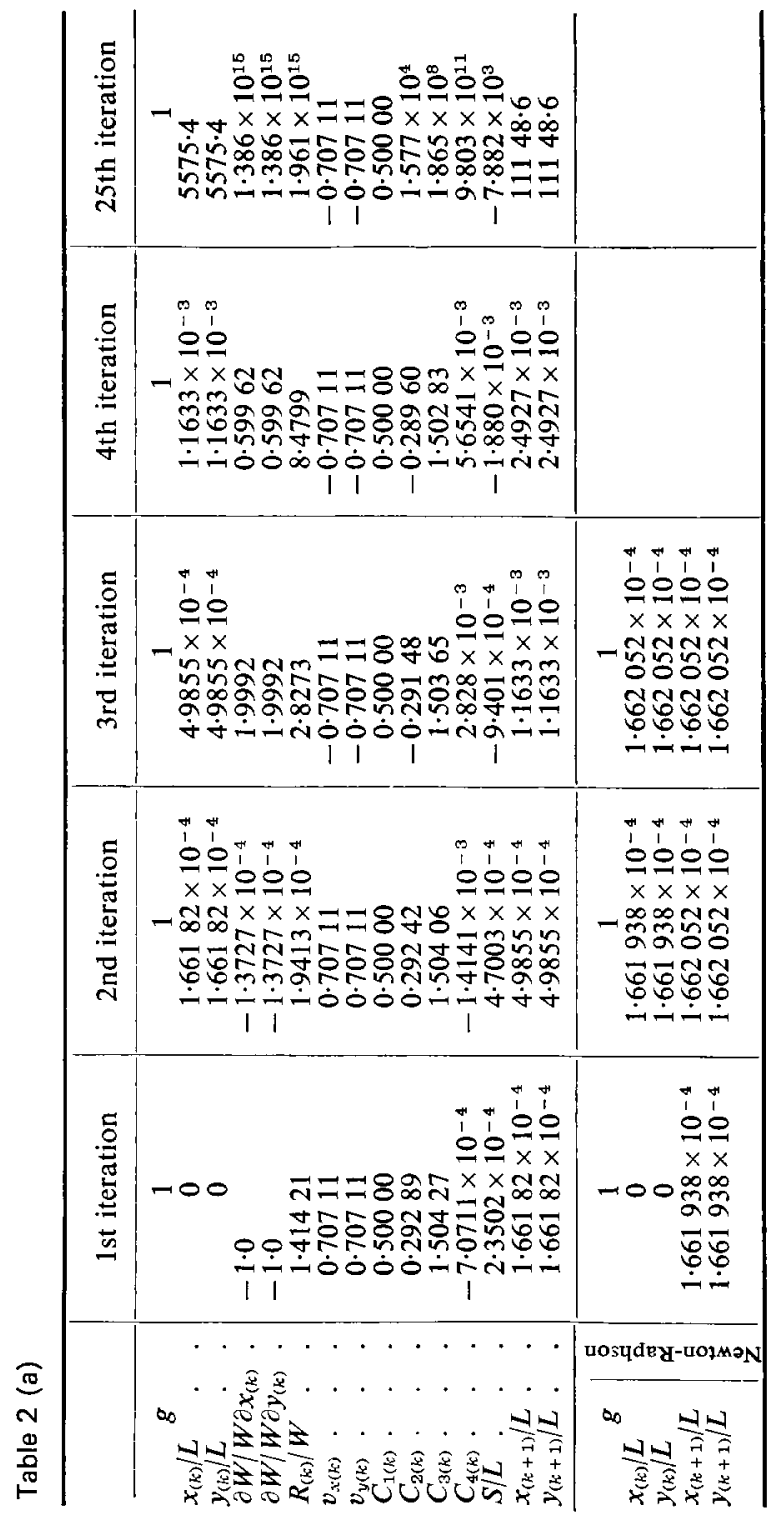




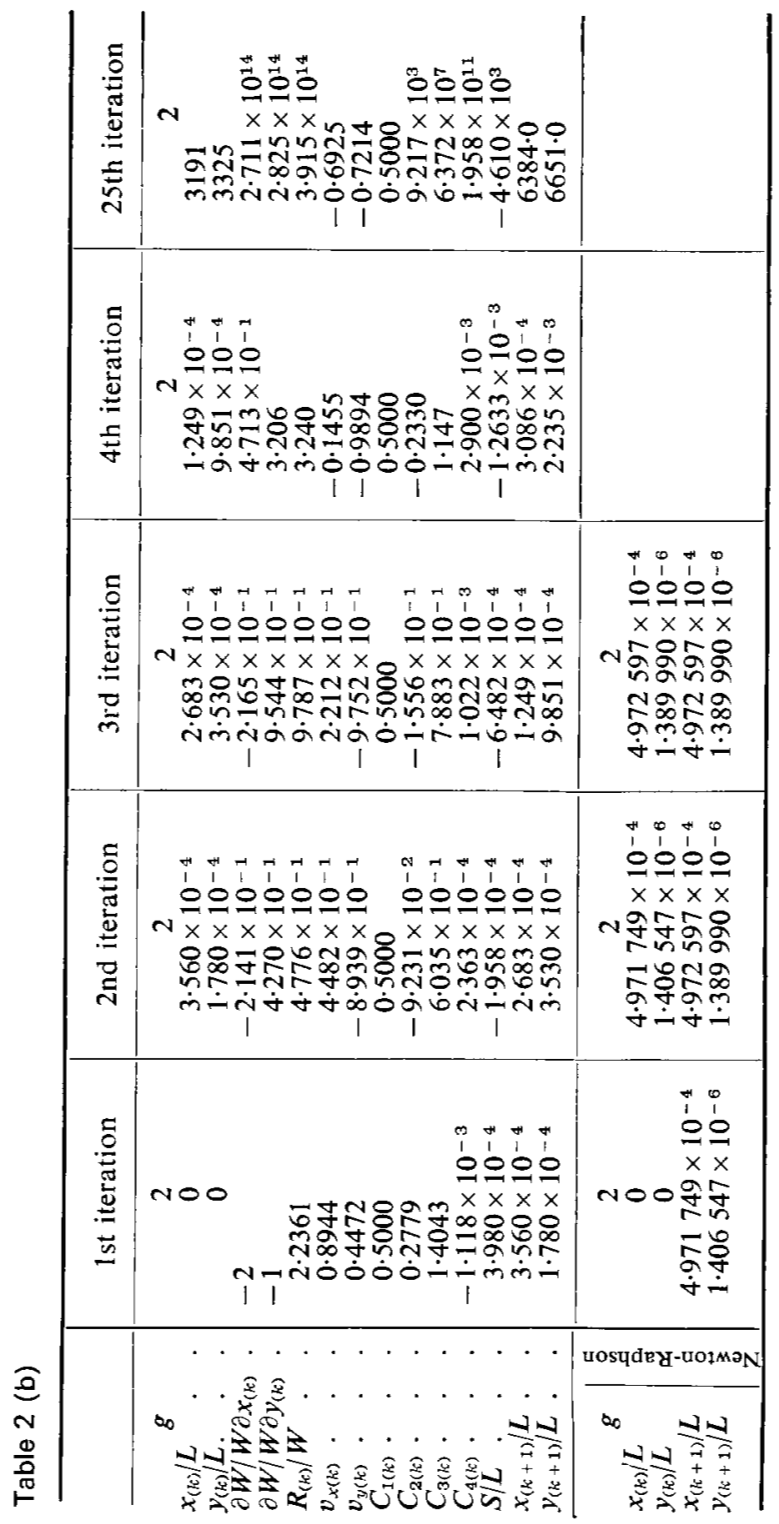


the matrix reduces to the ordinary stiffness matrix plus simple additional terms, in the structure $x$ and $y$ directions, dependent on the current values of the member axial loads; Brotton ${ }^{11}$ has also derived this simplified form but by physical reasoning. The non-linear program, using this simplified tangent stiffness matrix, was developed without difficulty from an existing linear plane frame analysis program by adding the additional terms into its stiffness matrix and introducing an iteration control. Any inaccuracies arising from the omission of the small order terms may normally be made negligible by adequate subdivision of flexural members.

79. I have used the program described for the analysis of the Author's example. More efficient running would have been obtained, of course, by rewriting the program omitting flexural terms; I consider, however, that a more useful comparison has been made by applying it in its general form. Actual analysis time on a CDC 3300 computer was one second per cycle of iteration and a complete run including program loading, data reading, analysis of three loading conditions, each being allowed five cycles of iteration, and printing of the results for three of the cycles of each loading condition, took just over one minute. Convergence errors were less than $0.01 \%$ after five cycles even with the heaviest load ( $W=10000 \mathrm{lbf} /$ joint). All values of displacements and member forces lay on the curves plotted by the Author in Figs 5-8, although a detailed comparison with the figures in Table 1 for $W=10000$ indicates differences of $0.1-0.5 \%$. It is likely that this discrepancy is caused by two approximations in the Author's theory. The first is the omission of terms including $e_{j n}{ }^{2}$ in the derivation of equation (14), one result of which is that equations (17) do not exactly represent unbalanced forces at the joints; the second is the use of the pretensioned lengths of members rather than the unstrained lengths as the zeroes from which strains are measured. The effect in this example is, as seen, insignificant; this may not be so for materials with a lower modulus of elasticity.

80. I have checked the numerical stability of the general finite deflexion program by applying loads $W=1000000 \mathrm{Ibf} /$ joint. The magnitude of the deflexions is indicated by the dotted lines in Fig. 10. Convergence errors were less than $0.01 \%$ in 10 cycles; with the exception of the forces in some of the strut members, errors of less than $1 \%$ were obtained in 8 cycles.

81. There are, however, two types of structure for which this method may fail. The first is a structural mechanism in the absence of pretension when the structure has no stiffness for the first cycle of iteration. This may be corrected, however, by inserting light springs at the nodes for the first cycle, and subsequently removing them. The second type is indicated in principle in Fig. 11, if the load is of sufficient magnitude to cause 'snap through'. In this case the additional term in the tangent stiffness matrix may cause negative stiffness at the apex at some stage of the calculation. It appears that the Author's method should succeed in both these cases without modification.

82. It is desirable that a non-linear analysis program of this type should be able to handle a structure in which the individual members have a non-linear force displacement curve. In principle there is no difficulty including this in the method I have outlined, and I should like to know whether the Author has attempted it in his

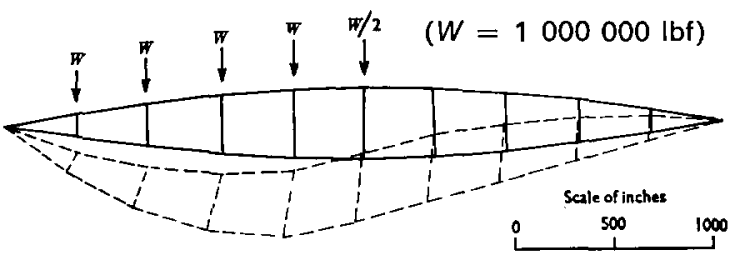

Fig. 10. Deflected form of structure

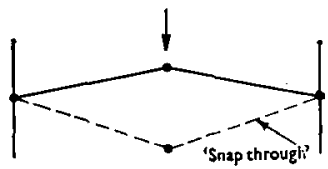

Fig. 11 
method. If, however, the curve is non-elastic, the difficulties appear to be formidable in either method.

83. Summarizing, therefore, the method I have adopted has the advantage of greater generality at the expense of somewhat reduced efficiency in particular applications and possible failure in certain unlikely circumstances. As an indication of its generality, it has been used successfully for analysis of such widely different structures as a large suspension bridge and a bent strut carrying an axial load equal to $75 \%$ of its Euler load. The Author's method has the advantage of high efficiency for particular applications and in its unexacting demands on computer hardware. Does the Author think this is a fair comparison?

\section{Dr H. A. Buchholdt}

I should like to thank Dr Poskitt and Mr Brown for their discussion and criticism of the Paper.

85. Dr Poskitt's points concerning the constant term in equation (25) and the use of infinitesimals are valid regarding notation. They do not, however, affect in any way the substance or details of the Author's argument. The remainder of his contribution is without foundation. With respect to the rectitude of equation (14), consider a link whose ends are initially at $(0,0)$ and $(5,0)$ and are finally at $(0,0)$ and (4.004, 3.003). The extension calculated from (14) is $e=0.0050025$, showing a proportional error of precisely the magnitude $e / 2 L$. If Dr Poskitt's suggestion were followed one would obtain $e=-0.996$, a value -198 times the correct result. The attempt to demonstrate instability is misconceived. In the first place the operations alleged to be a source of numerical difficulty consist merely in the normalization of a vector-always a well-conditioned process - and in the second place the normalization is not essential. It is precisely equivalent to a change of scale in $S$, and is used only in order that the coefficients $C_{1}, C_{2}$ and $C_{3}$ in equation (21) should remain always within a few orders of magnitude. The stability of any descent method is readily proved provided that the assembly itself is stable. (This last condition ensures that $C_{3}=\left[d^{2} W / d S^{2}\right]_{s=0} \lessgtr 0$.) Incremental displacements must, because they are in a direction of decreasing potential, always be directed to the inside of a closed surface in configuration space. Moreover, each step must terminate within that surface, because the step length is chosen so that the nearest minimum along the current step vector provides the next approximation. The closed surface on which the next step starts is therefore contained within the previous surface, and itself contains the solution point sought. It is observed that the constant $C_{4}=[d W / d S]_{s=0}$ and is thus the component of the gradient vector in the direction of $\left(v_{(k)}\right)$. From the definition of $\left(v_{(k)}\right)$ it follows that

$$
C_{4}=\left[\frac{\partial W}{\partial x_{(k)}}\right]^{T}\left[v_{(k)}\right]=-\sqrt{\left[\frac{\partial W}{\partial x_{(k)}}\right]^{T}\left[\frac{\partial W}{\partial x_{(k)}}\right]} \cdot \cdot . \quad . \quad .
$$

so that $C_{4}$, which approaches zero with approach to equilibrium, should invariably be negative. However, the fourth of equations (26) indicates that $C_{4}$ is actually computed as the difference between two possibly quite large numbers, and may be of poor accuracy as a result. (It is important to observe that this possibility of error has sources in no way resembling those asserted by Dr Poskitt.) The difficulty is completely eradicated by the use of equation (34) in place of the fourth of equations (26). Even when $C_{4}$ is subject to these difficulties the descent method cannot diverge. As soon as the unbalanced forces increase sufficiently to outweigh the round-off errors the method resumes convergence to the same solution as before. In practice it has been found that sign errors in $C_{4}$ do not arise until the absolute value of $C_{4}$ has lost all practical significance, and that the detection of a reversal of sign is a useful signal for the termination of the calculation. To proceed beyond this point is merely to generate answers whose apparent accuracy exceeds that of the data. 


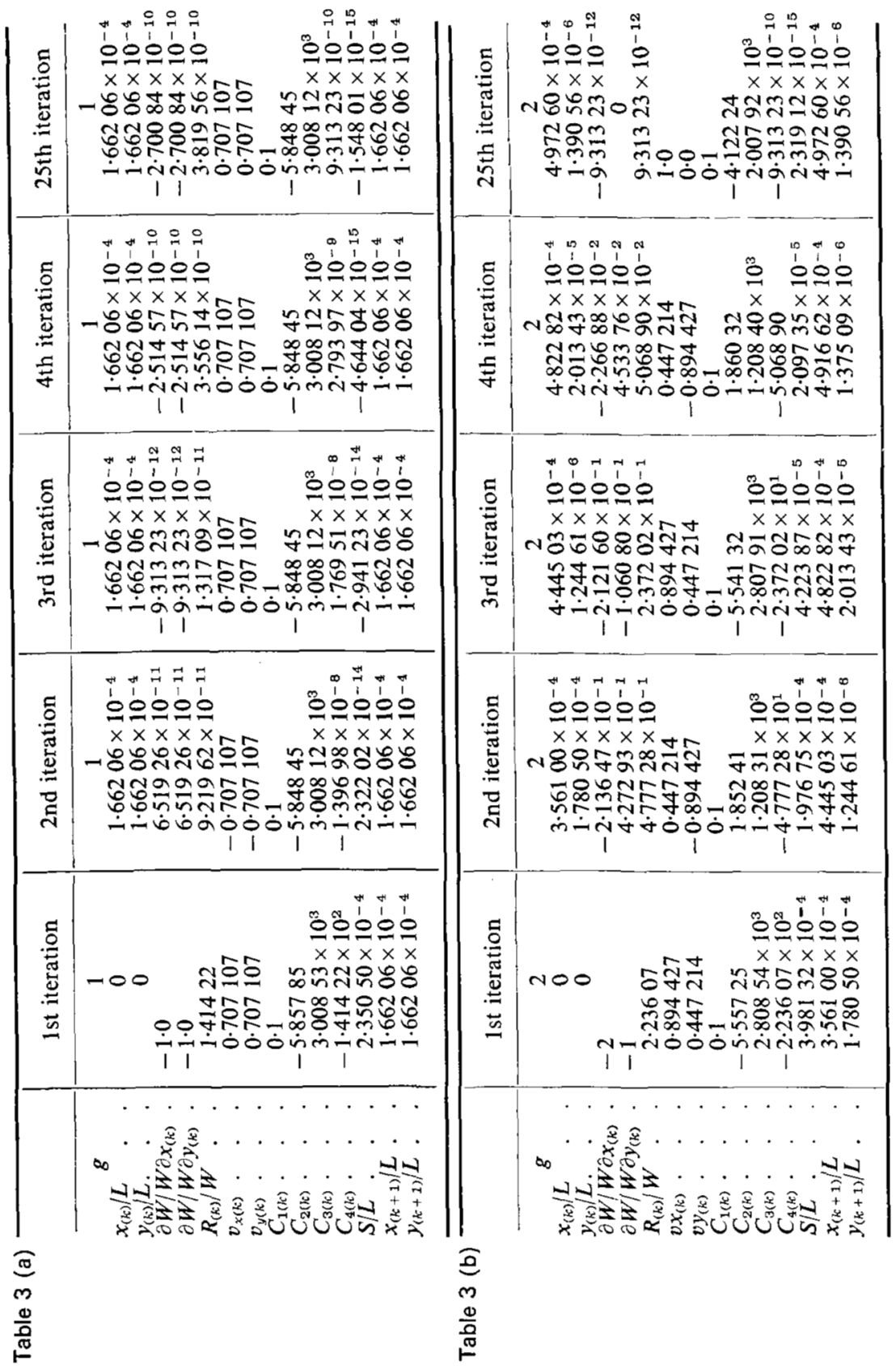


86. Dr Poskitt's numerical example (which is effectively linear and is, for reasons given, of little use for comparing methods) has merely been miscalculated. In particular, for his case $g=1$, the first descent vector actually passes through the solution point. The accurate solution of the cubic equation locates the solution in one step. Tables 3 (a) and (b) reproduce, in Dr Poskitt's format, the results of correct calculations for his examples. There is no trace of instability, although the remarks regarding the sign and value of $C_{4}$ are clearly illustrated.

87. In order to discuss the contributions advocating the Newton-Raphson method it is useful to clarify a few points. When attention is confined to stable assemblies the Newton-Raphson method is itself a descent method. Its major features are the extensive calculations aimed at finding an optimum descent direction, the complete omission of an exact determination of step length and the fact that for quadratic functions the minimum is reached in a single step (second order convergence). The descent direction is chosen with attention not only to the current gradient vector, but also to the current stiffness of the assembly. This involves setting up and solving a possibly very large system of linear equations, and the cost and accuracy of this are vital elements in any comparison. The solution of the linear equations is greatly affected by the condition of their matrix and it is easily shown that, for structures whose DMF is zero, no difficulties need be expected. For a structural mechanism the DMF is equal to the number of eigenvalues of the Newton-Raphson matrix whose order of magnitude is $P / L$, where $P$ is the tension in a typical member and $L$ is its length. The other eigenvalues are of order $E A / L$. Thus there are a number of eigenvalues whose ratio to the larger ones is of the order of the strain in a member. These can lead to condition troubles. The importance of this lies in the fact that, while the Newton-Raphson descent vector will in general be directed to the interior of a closed potential surface, the omission of any explicit calculation of its length admits the possibility that a step will be taken which emerges from the other side of the surface, so that instability can result. An obvious example occurs in the first step of the solution of a flat laterally-loaded cable net with no prestress. The Newton-Raphson matrix is singular $(P / L=0$ for each of DMF eigenvalues) and the step incalculable by that method, although the assembly is stable. Analogues of this situation can occur in complicated problems.

88. It is worth noticing that if the example given in the Paper is attempted to be solved by the Newton-Raphson method for $W=1000000 \mathrm{lbf} / \mathrm{joint}$, the calculated resultant step-lengths must be reduced as otherwise the method will diverge. Again it is worth noticing that Mr Brown refers to the use of an iteration control.

89. Evidently, if an assembly has $\mathrm{DMF}=0$ and a comparatively low number of degrees of freedom (or if the Newton-Raphson matrix is narrowly-banded), the Newton-Raphson method can be applied with confidence and reasonable economy. Such structures will usually be only weakly non-linear. For extensive structures and structural mechanisms the stability and economy of less intricate descent methods are desirable. It appears that, for the example given in the Paper, the Newton-Raphson method, converging in ten iterations, requires roughly twice the computer time needed by an alternative efficient descent method. Considerable effort and expense would be needed to provide definitive answers as to the best method for any particular problem.

90. Finally, for two dimensional systems, the theory in the Paper has been extended to include flexural members. The extended theory has, however, not yet been programmed. A programme for the analysis of pretensioned cable systems in which the load/extension curves for the members are non-linear is currently being developed.

\section{References}

7. Buckingham R. A. Numerical methods. Pitman, 1962.

8. Poskitt T. J. Numerical solution of nonlinear structures. Proc. Am. Soc. civ. Engrs, 1967, 93 (Aug.) ST4, 69-94. 
DISCUSSION

9. SAAfAn S. A. and Brotton D. M. Elastic finite deflection analysis of rigid frameworks by digital computer. Symposium on the use of computers in civil engineering, Lisbon, October 1962.

10. Tezcan S. S. Discussion on numerical solution of nonlinear structures. J. struct. Div. Am. Soc. civ. Engrs, 1968, 94 (June) ST6.

11. Brotton D. M. A general computer programme for the solution of suspension bridge problems. Struct. Engr, 1966 (May). 